CLINICAL STUDY

\title{
Cardiovascular haemodynamics and cardiac autonomic control in patients with subclinical and overt hyperthyroidism
}

\author{
Mario Petretta, Domenico Bonaduce, Letizia Spinelli, Maria L E Vicario, Vincenzo Nuzzo ${ }^{1}$, Fortunato Marciano ${ }^{2}$, \\ Pietro Camuso $^{1}$, Valerio De Sanctis and Giovanni Lupoli ${ }^{1}$ \\ Department of Internal Medicine, Cardiology and Heart Surgery, ${ }^{1}$ Department of Molecular and Clinical Endocrinology and Oncology, 'Federico II' \\ University of Naples and ${ }^{2}$ Institute of Cybernetics, National Research Council, Naples, Italy \\ (Correspondence should be addressed to D Bonaduce, Via A. Falcone 394, 80127-Naples, Italy; Email: bonaduce@unina.it)
}

\begin{abstract}
Objective: To characterize cardiac structure and function and cardiac autonomic control in patients with subclinical and overt hyperthyroidism.

Design: Thirty patients with subclinical hyperthyroidism and 30 with overt disease were selected from patients never previously treated for endocrinological disease in the outpatient clinic of our institution. Twenty normal individuals were studied as control group.

Methods: Left ventricular structure and function and cardiac autonomic control were evaluated, respectively, by two-dimensional Doppler echocardiography and by 24-h Holter recording with heart rate variability analysis.

Results: Patients with overt hyperthyroidism showed greater values of left ventricular end-diastolic volume $(P<0.05)$ and left ventricular mass $(P<0.05)$ than patients with subclinical disease. In addition, the mean velocity of left ventricular fibre shortening $(P<0.05)$ and left ventricular ejection fraction $(P<0.05)$ were greater in patients with overt hyperthyroidism than in patients with subclinical disease. No difference in any of these parameters was detectable between normal subjects and patients with subclinical disease. The isovolumic relaxation period was shorter in patients with subclinical hyperthyroidism than in control individuals $(P<0.05)$ and in patients with overt hyperthyroidism $(P<0.05)$. As regards cardiac autonomic control, all time and frequency domain measures decreased progressively from control individuals to patients with subclinical hyperthyroidism and those with overt disease $(P<0.001)$.

Conclusions: Thyrotoxic patients show changes in left ventricular structure and increased echocardiographic indexes of myocardial contractility, whereas the only echocardiographic feature detectable in patients with subclinical hyperthyroidism is an increased velocity of left ventricular relaxation. Cardiac parasympathetic withdrawal is evident in patients with overt hyperthyroidism and in patients with subclinical disease.
\end{abstract}

European Journal of Endocrinology 145 691-696

\section{Introduction}

Increased resting heart rate is one of the most common features in hyperthyroidism (1). Increased intrinsic heart rate (2) and increased sympathetic autonomic activity and reduced parasympathetic tone (3) may contribute to the genesis of sinus tachycardia. The similarities between the clinical manifestations of hyperthyroidism and of adrenergic hyperactivity have stimulated intensive study of the possible interactions between thyroid hormones and the sympathetic nervous system. Cacciatori et al. (4) studied cardiac autonomic function in thyrotoxicosis and found a reduced parasympathetic activity with a hypersympathetic tone in untreated hyperthyroid patients. Differently, it has been suggested that the increase in blood pressure and in cardiac output resulting from thyrotoxicosis may induce a reflex vagal activation and sympathetic inhibition (5). In the present study we utilized two-dimensional Doppler echocardiography and power spectral analysis of the heart rate to characterize cardiovascular haemodynamics and cardiac autonomic control in patients with subclinical hyperthyroidism and in patients with overt hyperthyroidism.

\section{Methods}

\section{Study participants}

The overall study population included 30 patients with subclinical hyperthyroidism, 30 patients with overt hyperthyroidism and 20 normal individuals. The 
investigation conformed to the recommendations of the Declaration of Helsinki and the study was approved by the ethics committee of our institution. All participants gave written informed consent before entering the study. The patients were selected from patients never previously treated for endocrinological disease in the outpatient clinic of our institution. Subclinical hyperthyroidism was caused by Graves' disease in six patients, autonomous adenoma in 14 and multinodular goitre in 10 patients. Overt hyperthyroidism was caused by Graves' disease in seven patients, autonomous adenoma in 15 and multinodular goitre in eight. For the purpose of this study, we excluded patients in whom hyperthyroidism was due to an excess of L-thyroxine. Exclusion criteria were any other major disease, ischaemic heart disease, hypertension, diabetes, atrial fibrillation or severe ventricular arrhythmia, and cardiothoracic anatomy not allowing for satisfactory and reproducible echocardiographic recordings.

\section{Measurement of hormones}

Thyroid hormones and thyrotropin (TSH) were measured by using an immunoradiometric assay (Radim (Pomezia, Rome, Italy): intra-assay coefficient of variability $6.6 \%$, normal reference interval $0.3-$ $3.5 \mathrm{mIU} / \mathrm{l})$. Serum concentrations of free tri-iodothyronine $\left(\mathrm{FT}_{3}\right)$ and free thyroxine $\left(\mathrm{FT}_{4}\right)$ were determined by radioimmunoassay (RTA kit, Lisophase kit (Technogenetics, Milan, Italy); intra-assay coefficient of variability $3.8 \%$ for $\mathrm{FT}_{3}$ and $4.9 \%$ for $\mathrm{FT}_{4}$; normal reference interval for $\mathrm{FT}_{3}$ and $\mathrm{FT}_{4}: 1.6-3.4 \mathrm{pg} / \mathrm{ml}$ and $7.1-$ $18.5 \mathrm{pg} / \mathrm{ml}$, respectively). Subclinical hyperthyroidism was diagnosed by normal serum $\mathrm{FT}_{3}$ and $\mathrm{FT}_{4}$ concentrations and decreased serum TSH concentrations $(<0.3 \mathrm{mIU} / \mathrm{l})$. Overt hyperthyroidism was diagnosed clinically and confirmed by high serum free $\mathrm{T}_{4}$ concentrations with suppressed serum TSH concentrations $(<0.1 \mathrm{mIU} / \mathrm{l})$. Clinical characteristics and thyroid hormone serum concentrations of controls and of patients with subclinical and overt hyperthyroidism are reported in Table 1. Study patients and control subjects underwent echocardiographic examination and 24-h Holter recording on two consecutive days. Holter recording started between $0800 \mathrm{~h}$ and $1000 \mathrm{~h}$. After this monitoring was terminated, a complete echocardiographic and Doppler examination was performed.

\section{Echocardiographic and Doppler measurements}

Wide-angle, two-dimensional echoes were recorded with a phased-array sector scanner (Sonos 2000, Hewlett-Packard Co., Andover, MA, USA). The left ventricular long axis $\left(\mathrm{L}_{\max },\right)$ was measured at enddiastole as the longest major axis in either of the two apical views: four chamber view and two chamber view. The measurements of $\mathrm{L}_{\max }$ were rounded off to the nearest whole number to ensure reproducibility. Left ventricular end-diastolic area (EDA) was measured using the largest of all the left ventricular minor axes measured. Left ventricular end-diastolic volume (EDV), in millilitres, was calculated according to the singleplane ellipse method as EDV $=8 / 3\left(\mathrm{EDA}^{2}\right) /\left(\pi \times \mathrm{L}_{\max }\right)$ (6). The same measurements were taken at end-systole to calculate end-systolic volume (ESV). Left ventricular ejection fraction (LVEF) was measured using the averages of all the EDV and ESV values. All studies were performed by the same investigator and read independently by two experts unaware of the procedure; their readings of both $\mathrm{L}_{\max },(r=0.97 ; P<$ $0.001)$ and EDA $(r=0.96 ; P<0.001)$ were closely correlated and their measurements of EDV $(r=0.97$; $P<0.001)$ and ESV $(r=0.95 ; P<0.001)$ were also in excellent correlation. The inter-observer variability of several measurements of volumes over a period of $2 \mathrm{~h}$ did not exceed $3.5 \%$.

Left ventricular mass was calculated according to the Penn convention (7) and was corrected for body surface area and expressed in $\mathrm{g} / \mathrm{m}^{2}$. The left ventricular enddiastolic and end-systolic diameters (LVEDD and LVESD), interventricular septal thickness (IST) and

Table 1 Characteristics of the study population.

\begin{tabular}{lcccr}
\hline & $\begin{array}{c}\text { Controls } \\
(n=20)\end{array}$ & $\begin{array}{c}\text { Subclinical hyperthyroidism } \\
(n=30)\end{array}$ & $\begin{array}{c}\text { Overt hyperthyroidism } \\
(n=30)\end{array}$ & P \\
\hline Age (years) & $47 \pm 9$ & $48 \pm 10$ & $49 \pm 10$ & $\mathrm{NS}$ \\
Women & $16(80)$ & $25(84)$ & $24(80)$ & $\mathrm{NS}$ \\
SBP $(\mathrm{mmHg})$ & $121 \pm 9$ & $123 \pm 12$ & $124 \pm 11$ & $\mathrm{NS}$ \\
DBP $(\mathrm{mmHg})$ & $78 \pm 6$ & $82 \pm 5$ & $85 \pm 6$ & $\mathrm{NS}$ \\
TSH $(\mathrm{mlU} / \mathrm{l})$ & $2.6 \pm 0.92$ & $0.05 \pm 0.03^{*}$ & $0.002 \pm 0.01^{*} \dagger$ & $<0.001$ \\
$\mathrm{FT}_{3}(\mathrm{pg} / \mathrm{ml})$ & $2.6 \pm 0.51$ & $2.9 \pm 0.41$ & $6.1 \pm 0.81^{*} \dagger$ & $<0.001$ \\
$\mathrm{FT}_{4}(\mathrm{pg} / \mathrm{ml})$ & $12 \pm 2.9$ & $13 \pm 3.8$ & $27 \pm 5.7^{*} \dagger$ & $<0.001$ \\
\hline
\end{tabular}

Values are means \pm S.D. or number (\%). SBP, DBP, systolic and diastolic blood pressures; $\mathrm{TSH}$, thyroid stimulating hormone; $\mathrm{FT}_{3}$, free tri-iodothyronine; $\mathrm{FT}_{4}$, free thyroxine.

NS, not significant; ${ }^{\star} P<0.05$, compared with controls; $† P<0.05$, overt hyperthyroidism compared with subclinical hyperthyroidism. 
posterior wall thickness (PWT) were measured according the recommendations of the American Society of Echocardiography (8). The left ventricular percent fractional shortening $(\% \Delta \mathrm{D})$ was calculated as (LVEDD-LVESD)/LVEDD. We also calculated the left ventricular ejection time (LVET, in ms) (9), the ratecorrected left ventricular mean velocity of fibre shortening expressed as circumferences (VCFc, in circ/s) (10) and the isovolumic relaxation period (IRP, in ms) (11). Recordings of mitral inflow velocity were made from an apical four-chamber view. From the Doppler spectral tracing, we calculated the peak velocity of early diastolic rapid inflow (peak $\mathrm{E}$, in $\mathrm{cm} / \mathrm{s}$ ), the peak velocity of atrial contraction (peak $\mathrm{A}$, in $\mathrm{cm} / \mathrm{s}$ ) and their ratio $(\mathrm{E} / \mathrm{A})$.

\section{Processing 24-h Holter recordings}

All 24-h Holter recordings were analysed at the National Research Council cybernetics laboratory as previously described $(12,13)$. The two electrocardiographic analog channels were read via a modified TeacTascam 234 Syncaset tape deck (Teac Corporation, Tokyo, Japan) and digitized at 330 samples/s. All R-R interval sequences were stored, and each was labeled with a code number identifying its normality or class of abnormality. Premature complexes and their adjacent $\mathrm{R}-\mathrm{R}$ intervals, used only for timekeeping purposes, were rejected by the software, as were electrical noise and other aberrant electrocardiogram (ECG) signals. The sequence of normal $\mathrm{R}-\mathrm{R}(\mathrm{N}-\mathrm{N})$ intervals was analysed to compute time and frequency domain measures of heart rate variability (HRV) (14).

\section{Time domain measures of HRV}

The standard deviation of the $\mathrm{N}-\mathrm{N}$ intervals (SDNN) calculated over a 24-h period encompasses both shortterm and long-term $\mathrm{N}-\mathrm{N}$ interval variations. The SD of the average $\mathrm{N}-\mathrm{N}$ intervals for all 5-min segments of the entire 24-h ECG recording (SDANN index) also evaluates long-term $\mathrm{R}-\mathrm{R}$ variations, whereas the mean of the SDs of $\mathrm{N}-\mathrm{N}$ intervals for all 5-min segments of the entire 24-h ECG recording (SDNN index) depends on short-term $\mathrm{R}-\mathrm{R}$ variations. In contrast, differences between successive $\mathrm{N}-\mathrm{N}$ intervals provide an index of cardiac vagal modulation that is firmly related to short-term variations in heart rate. Accordingly, we calculated the root mean square successive difference ( $\mathrm{r}-\mathrm{MSSD}$ ) of all $\mathrm{N}-\mathrm{N}$ intervals and the percentage of differences between adjacent $\mathrm{N}-\mathrm{N}$ intervals exceeding $50 \mathrm{~ms}$ (pNN50) for the entire 24-h recordings.

\section{Frequency domain measures of HRV}

The 24-h heart rate power spectrum was computed by means of the fast-Fourier transform algorithm, as previously described $(12,13)$. The final average spectrum was expressed for each band as milliseconds squared. The bands explored were very-low-frequency power (the energy in heart rate power spectrum between 0.0033 and $0.04 \mathrm{~Hz}$ ), low-frequency (LF) power (between 0.04 and $0.15 \mathrm{~Hz}$ ) and high-frequency (HF) power (between 0.15 and $0.40 \mathrm{~Hz}$ ). The ratio of LF to HF power was calculated from the absolute values of these two components.

\section{Statistical analysis}

Statistical analysis was performed using the SPSS statistical package (15). Categorical variables were expressed as percentage and compared using the $\chi^{2}$-test. Continuous data were expressed as mean \pm SD and compared using one-way analysis of variance; if a significant F-value was found, then multiple comparisons were performed with the Bonferroni correction. Finally, to evaluate whether it was possible to differentiate between cardiac parameters of patients with hyperthyroidism caused by autoimmune Graves' disease and those of hyperthyroidism caused by nonimmune autonomous thyroid nodules, we performed a separate subgroup analysis of patients with hyperthyroidism of different origin. Because the distribution of the frequency domain measures of heart rate variability were extremely skewed, the log transformation $(\ln )$ of each measure, which produces nearly normal distributions, was applied before statistical analysis was performed.

\section{Results}

\section{Echocardiographic data}

Echocardiographic data of control individuals and of patients with subclinical and overt hyperthyroidism are reported in Table 2. As shown, left ventricular enddiastolic volume (LVEDV) and left ventricular mass index (LVMI) were greater in patients with overt hyperthyroidism than in the other two groups. In addition, VCF, rate-corrected VCF (VCFc), and LVEF were greater in patients with overt hyperthyroidism than in controls and in patients with subclinical disease. There was no difference between control subjects and patients with subclinical disease in any of these parameters. As regards IRP, this parameter was lower in those with subclinical or overt hyperthyroidism than in control individuals. Furthermore, it was slightly shorter in subclinical hyperthyroidism than in overt disease. Finally, the E/A ratio was lower in patients with overt hyperthyroidism than in the other two groups, but no difference was detectable between normal individuals and patients with subclinical hyperthyroidism. On subgroup analysis performed separately in patients with subclinical and overt hyperthyroidism, no difference was detectable in 
Table 2 Echocardiographic and Doppler data.

\begin{tabular}{lcccc}
\hline & $\begin{array}{c}\text { Controls } \\
(n=20)\end{array}$ & $\begin{array}{c}\text { Subclinical hyperthyroidism } \\
(n=30)\end{array}$ & $\begin{array}{c}\text { Overt hyperthyroidism } \\
(n=30)\end{array}$ & P \\
\hline Left atrium (mm) & $32 \pm 2$ & $32 \pm 3$ & $34 \pm 2$ & $\mathrm{NS}$ \\
Aortic root (mm) & $32 \pm 2$ & $32 \pm 3$ & $32 \pm 3$ & $\mathrm{NS}$ \\
LVMI (g/m $\left.\mathrm{m}^{2}\right)$ & $97 \pm 5$ & $104 \pm 11$ & $120 \pm 10^{*} \dagger$ & $<0.001$ \\
LVEDV (ml) & $104 \pm 10$ & $113 \pm 13$ & $41 \pm 13^{*} \dagger$ & $<0.001$ \\
LVESV (ml) & $43 \pm 5$ & $44 \pm 7$ & $65 \pm 3^{*} \dagger$ & $<0.01$ \\
LVEF (\%) & $58 \pm 4$ & $62 \pm 3$ & $267 \pm 13^{*} \dagger$ & $<0.001$ \\
LVET (ms) & $303 \pm 20$ & $296 \pm 35$ & $1.79 \pm 0.25^{*} \dagger$ & $<0.001$ \\
VCF (circ/s) & $1.40 \pm 0.15$ & $1.51 \pm 0.24$ & $1.27 \pm 0.17^{*} \dagger$ & $<0.001$ \\
VCFc (circ/s) & $1.11 \pm 0.19$ & $1.13 \pm 0.19$ & $90 \pm 4^{*} \dagger$ & $<0.001$ \\
IRP (ms) & $96 \pm 5$ & $85 \pm 7^{*}$ & $1.02 \pm 0.28^{*} \dagger$ & $<0.001$ \\
E/A & $1.44 \pm 0.27$ & $1.10 \pm 0.33$ & $<0.01$
\end{tabular}

Values are means \pm S.D. LVMI, left ventricular mass index; LVEDV, left ventricular end-diastolic volume; LVESV, left ventricular end-systolic volume; LVEF, left ventricular ejection fraction; LVET, left ventricular ejection time; VCF, left ventricular mean velocity of fibre shortening; VCFc, rate-corrected mean shortening velocity; IRP, isovolumic relaxation period; $E / A$, ratio of peak velocity of early distolic filling (E) to peak velocity of atrial contraction (A).

${ }^{*} P<0.05$ compared with contols; $\dagger P<0.05$ overt hyperthyroidism compared with subclinical hyperthyroidism (ANOVA).

echocardiographic data between patients with hyperthyroidism caused by either Graves' disease or autonomous nodules.

\section{HRV analysis of 24-h Holter recordings}

Results of heart period variability analysis obtained from 24-h Holter recordings are reported in Table 3. Average N-N intervals and all time- and frequencydomain measures decreased progressively from normal individuals to patients with subclinical hyperthyroidism and patients with overt disease. Interestingly, all HRV measures were lower in patients with overt hyperthyroidism than in those with subclinical illness. The $\mathrm{LF}$ to $\mathrm{HF}$ power ratio, comparable between control individuals and patients with subclinical hyperthyroidism, was greater in patients with overt hyperthyroidism. No difference was detectable in HRV measures between patients with hyperthyroidism caused by Graves' disease or by autonomous nodules.

\section{Discussion}

Our study demonstrated that patients with overt hyperthyroidism had increased left ventricular mass and contractility and an unbalanced cardiac autonomic control with parasympathetic withdrawal. The parasympathetic withdrawal was also detectable in patients with subclinical hyperthyroidism but, in this group, cardiac structure and contractility remained

Table 3 Heart rate variability measures by $24-\mathrm{h}$ Holter recording.

\begin{tabular}{|c|c|c|c|c|}
\hline & $\begin{array}{l}\text { Controls } \\
(n=20)\end{array}$ & $\begin{array}{l}\text { Subclinical hyperthyroidism } \\
\qquad(n=30)\end{array}$ & $\begin{array}{l}\text { Overt hyperthyroidism } \\
\qquad(n=30)\end{array}$ & $\boldsymbol{P}$ \\
\hline \multicolumn{5}{|c|}{ Time domain measures } \\
\hline Average $\mathrm{N}-\mathrm{N}(\mathrm{ms})$ & $793 \pm 94$ & $746 \pm 62^{*}$ & $712 \pm 47^{*}$ & $<0.001$ \\
\hline SDNN (ms) & $139 \pm 32$ & $114 \pm 32^{*}$ & $108 \pm 26^{*}$ & $<0.001$ \\
\hline SDANN index (ms) & $123 \pm 31$ & $102 \pm 31^{*}$ & $98 \pm 14^{*}$ & $<0.001$ \\
\hline SDNN index (ms) & $65 \pm 53$ & $22 \pm 12^{*}$ & $14 \pm 4^{*} \dagger$ & $<0.001$ \\
\hline r-MSSD (ms) & $39 \pm 6$ & $33 \pm 12^{\star}$ & $25 \pm 3^{*} \dagger$ & $<0.001$ \\
\hline pNN50 (\%) & $16 \pm 8$ & $10 \pm 9^{*}$ & $6 \pm 2^{*}+$ & $<0.001$ \\
\hline \multicolumn{5}{|c|}{ Frequency domain measures } \\
\hline VLF power $\left(\mathrm{ms}^{2}\right)$ & $1739 \pm 375$ & $1281 \pm 323$ & $1181 \pm 191$ & \\
\hline In VLF power & $7.44 \pm 0.20$ & $7.12 \pm 0.29^{\star}$ & $7.1 \pm 0.12^{*}$ & $<0.001$ \\
\hline LF power $\left(\mathrm{ms}^{2}\right)$ & $634 \pm 204$ & $521 \pm 145^{\star}$ & $445 \pm 85^{\star}$ & \\
\hline In LF power & $6.40 \pm 0.32$ & $6.21 \pm 0.32^{*}$ & $6.01 \pm 0.09^{*} \dagger$ & $<0.001$ \\
\hline $\mathrm{HF}$ power $\left(\mathrm{ms}^{2}\right)$ & $321 \pm 146$ & $279 \pm 91$ & $197.4 \pm 44$ & \\
\hline In HF power & $5.67 \pm 0.45$ & $5.58 \pm 0.34$ & $5.26 \pm 0.2^{*} \dagger$ & $<0.001$ \\
\hline $\mathrm{LF} / \mathrm{HF}$ & $2.11 \pm 0.37$ & $1.91 \pm 0.30$ & $2.36 \pm 0.41^{*} \dagger$ & $<0.001$ \\
\hline
\end{tabular}

Values are means \pm S.D. $\mathrm{N}-\mathrm{N}$, normal $\mathrm{R}-\mathrm{R}$ interval; SDNN, standard deviation of all $\mathrm{N}-\mathrm{N}$ intervals; SDANN index, S.D. of the average $\mathrm{N}-\mathrm{N}$ intervals for all 5-min segments; SDNN index, mean of the S.D. of $\mathrm{N}-\mathrm{N}$ intervals for all 5-min segments; r-MSSD, root mean square successive difference; pNN50, percentage of differences between successive $\mathrm{N}-\mathrm{N}$ intervals $>50 \mathrm{~ms}$; In, logarithmic units; VLF, very-low frequency; LF, low frequency; HF, high frequency; $\mathrm{LF} / \mathrm{HF}$, low to high frequency power ratio.

${ }^{*} P<0.05$ compared with controls; $\uparrow P<0.05$ overt hyperthyroidism compared with subclinical hyperthyroidism. 
normal and the reduction in IRP was the only echocardiographic feature detectable.

\section{Cardiac structure and function}

The thyrotoxic state induces profound alterations in the cardiovascular system, including an increase in total blood volume, a decrease in total systemic vascular resistance and shortened circulatory time. These haemodynamic changes cause an increase in cardiac work that leads, in time, to cardiac hypertrophy (16). Furthermore, thyroid hormones have been demonstrated to regulate genes that encode both structural and regulatory proteins in the heart, with an increase in myocardial contractility (17). In fact, in the heart thyroid hormones directly alter the expression of alpha-myosin heavy chain, which has greater ATPase activity than betamyosin heavy-chain $(18,19)$. In addition, production of the sarcoplasmic reticulum $\mathrm{Ca}^{2+}$-ATPase (SERCA2) and phospholamban is regulated by $\mathrm{T}_{3}$, acting through changes in gene transcription $(17,20)$. The thyroid hormone-induced changes in SERCA2 protein concentrations are inversely related to alteration in the concentrations of the SERCA2 inhibitor protein, phospholamban. On the basis of these observations, it has been suggested that thyroid hormone-mediated changes in the relative ratio of phospholamban to $\mathrm{Ca}^{2+}$-ATPase regulate the $\mathrm{Ca}^{2+}$ uptake rates by the reticulum and the relaxation properties of the myocardium (17). Release of calcium and its reuptake into the sarcoplasmic reticulum are critical determinants of systolic contractile function and diastolic relaxation, and explain the changes in contractility and the increased diastolic function in patients with hyperthyroidism (21). Moreover, recently an overexpression of functional ryanodine receptors in the hyperthyroid rabbit heart has been demonstrated (22). The relative abundance of ryanodine receptors may be responsible for the increase in $\mathrm{Ca}^{2+}$ released from sarcoplasmic reticulum to trigger muscle contraction, with an increase in myocardial contractility associated with thyroid hormone-induced cardiac hypertrophy (22). The increased values of LVMI, LVEF and VCFc observed in patients with hyperthyroidism are in agreement with these data. As regards diastolic function, thyroid hormones enhance the diastolic relaxation rate, which contributes to the increase in LVEDV (21). In our study, patients with hyperthyroidism showed a shortening of IRP that, interestingly, was more evident in patients with subclinical than in those with overt hyperthyroidism. Thus, in hyperthyroid patients with left ventricular hypertrophy, the increased velocity of relaxation may be offset by increased left ventricular stiffness. In addition, the reduction in E/A ratio observed in patients with hyperthyroidism may be a consequence of the increased left ventricular stiffness.

\section{Thyroid hormones and cardiac autonomic control}

The results of this study demonstrate a parasympathetic withdrawal in patients with overt hypertyroidism. In fact, HF and LF powers were lower in patients with overt hyperthyroidism than in control individuals. HF power is considered a marker of the modulation of vagal tone by the frequency and depth of ventilation $(23,24)$. LF power is a measure of the modulation of vagal and sympathetic tone by baroreflex activity; however, when evaluated utilizing the fast-Fourier transform of 24-h recordings, it is a greater reflection of parasympathetic activity (25). The ratio of LF power to HF power is considered to reflect the sympathovagal balance: high values suggest sympathetic predominance and low values sympathetic withdrawal (14). Therefore, the greater ratio in patients with clinical hyperthyroidism indicates a parasympathetic withdrawal. The lower values of r-MSSD and of percentage of difference between adiacent $\mathrm{R}-\mathrm{R}$ intervals $>50 \mathrm{~ms}-$ time-domain measures that also reflect vagal control confirm this finding.

Cacciatori et al. (4), utilizing power spectral analysis of heart rate, found a sharp reduction in HF components in hyperthyroid individuals compared with controls, with comparable values of LF components, and thus a high LF/HF ratio. In addition, Maciel et al. (3) found that a marked reduction in the efferent activity of the parasympathetic component was the main mechanism that modified resting heart rate in hyperthyroidism. Differently, Matsukawa et al. (26) found a significative negative correlation between the serum concentration of thyroid hormones and muscle sympathetic nerve activity. Hyperthyroidism increases the number and, potentially, the affinity of $\beta$-adrenergic receptors and also modifies the intracellular $G$ protein level subunits that activate adenylate cyclase, thus enhancing the transduction potential of agonist binding to adrenergic receptors $(27,28)$. The changes in adrenergic responsiveness may represent the primary effects of the abnormal concentrations of thyroid hormones, whereas the reduction in sympathetic outflow, as demonstrated by microneurography, may be a functional adaptation to such altered adrenergic responsiveness. In our study population, the aetiology did not influence the structural and functional cardiac features of both subclinical and overt hyperthyroidism. In fact, echocardiographic data and HRV measures were comparable in patients with Graves' disease and those with autonomous thyroid nodules.

It has been demonstrated that endogenous subclinical hyperthyroidism has a significant clinical impact and that it affects cardiac morphology and function $(29,30)$. Interestingly, we found a reduction in cardiac parasympathetic control in patients with subclinical hyperthyroidism. The mechanism of the reduction in cardiac vagal control in patients with suppressed TSH 
values despite $\mathrm{FT}_{3}$ and $\mathrm{FT}_{4}$ remaining in the normal range is not clear, but certainly contributes to symptoms.

\section{Conclusions}

Our study demonstrates that cardiac parasympathetic withdrawal is clearly evident in patients with overt hyperthyroidism and may also be recognized in patients with subclinical disease. This finding has important clinical implications and supports the hypothesis that treatment of patients with subclinical hyperthyroidism may prevent cardiac arrhythmias and improve the quality of life.

\section{References}

1 Gomberg-Maitland M \& Frishman WH. Thyroid hormone and cardiovascular disease. American Heart Journal $1998135187-$ 196.

2 Valcavi R, Menozzi C, Roti E, Zini M, Lolli G, Roti S et al. Sinus node function in hyperthyroid patients. Journal of Clinical Endocrinology and Metabolism 199275 239-242.

3 Maciel CB, Gallo L Jr, Marin Neto JA, Maciel LMZ, Alves MLD, Paccola GMF et al. The role of the autonomic nervous system in the resting tachycardia of human hyperthyroidism. Clinical Science 198772 239-244.

4 Cacciatori V, Bellavere F, Pezzarossa A, Dellera A, Gemma ML, Thomaset $\mathrm{K}$ et al. Power spectral analysis of heart rate in hyperthyroidism. Journal of Clinical Endocrinology and Metabolism $1996812828-2835$.

5 Safa-Tisseront V, Ponchon P, Laude D \& Elghozi JL. Autonomic contribution to the blood pressure and heart rate variability changes in early experimental hyperthyroidism. Journal of Hypertension 199816 1989-1992.

6 Folland ED, Parisi AF, Moghihan BS, Joney DR, Feldman CL \& Tow DE. Assessment of left ventricular ejection fraction and volumes by real-time, two-dimensional echocardiography. Circulation $197960760-771$.

7 Devereux RB \& Reicheck N. Echocardiographic determination of left ventricular mass in men. Circulation 198755 613-618.

8 Sahn DJ, DeMaria A, Kisslo J, Weyman A \& the Committee on Mmode Standardization of the American Society of Echocardiography. Recommendations regarding quantification in M-mode echocardiography: results of a survey of echocardiographic measurements. Circulation $1978 \mathbf{5 8} 1072-1081$.

9 Parisi AF, Hamilton BP, Thomas CN \& Mazzaferri EL. The short cardiac pre-ejection period. An index of thyrotoxicosis. Circulation 197449 900-904.

10 Colan SD, Borow KM \& Neumann A. The left ventricular endsystolic wall stress-velocity of fiber shortening relation: a loadindependent index of myocardial contractility. Journal of the American College of Cardiology 19844 715-724.

11 Spirito B, Maron BJ \& Bonow R. Noninvasive assessment of left ventricular diastolic function: comparative analysis of Doppler echocardiographic and radionuclide angiographic techniques. Journal of the American College of Cardiology 19867 518-526.

12 Bonaduce D, Marciano F, Petretta M, Migaux ML, Morgano G, Bianchi $V$ et al. Effects of converting enzyme inhibition on heart period variability in patients with acute myocardial infarction. Circulation 199490 108-113.

13 Bonaduce D, Petretta M, Ianniciello A, Apicella C, Cavallaro V \& Marciano F. Comparison of verapamil versus felodipine on heart rate variability after acute myocardial infarction. American Journal of Cardiology 199779 564-569.
14 Task Force of the European Society of Cardiology and the North American Society of Pacing and Electrophysiology. Heart rate variability. Standards of measurement, physiological interpretation, and clinical use. Circulation 199693 1043-1065.

15 Norusis, MJ. SPSS for Windows. Base System User's Guide. Release 6.0, pp 267-280. Chicago, Illinois: SPSS Inc., 1993.

16 Klein I \& Ojamaa K. Thyroid hormone and the cardiovascular system. New England Journal of Medicine 2001344 501-509.

17 Kiss E, Jakab G, Kranias EG \& Edes I. Thyroid hormone-induced alterations in phosholamban protein expression: regulatory effects on sarcoplasmic reticulum $\mathrm{Ca}^{2+}$ transport and myocardial relaxation. Circulation Research 199475 245-251.

18 Morkin E. Regulation of myosin heavy chain genes in the heart. Circulation 199387 1451-1460.

19 Ojamaa K, Klemperer JD, MacGilvray SS, Klein I \& Samarel A. Thyroid hormone and hemodynamic regulation of beta-myosin heavy chain promoter in the heart. Endocrinology 1996137 802-808.

20 Kimura Y, Otsu K, Nishida K, Kuzuya T \& Tada M. Thyroid hormone enhances $\mathrm{Ca}^{2+}$ ATPase and decreasing phospholamban expression. Journal of Molecular and Cellular Cardiology 199426 1145-1154.

21 Mintz G, Pizzarello R \& Klein I. Enhanced left ventricular diastolic function in hyperthyroidism: noninvasive assessment and response to treatment. Journal of Clinical Endocrinology and Metabolism 199173 146-150.

22 Jiang $\mathrm{M}$, Xu A, Tokmakejian S \& Narayanan N. Thyroid hormone-induced overexpression of functional ryanodine receptors in the rabbit heart. American Journal of Physiology. Heart and Circulatory Physiology 2000278 H1429-H1438.

23 Pagani M, Lombardi F, Guzzetti S, Rimoldi O, Furlan R, Pizzinelli P et al. Power spectral analysis of heart rate and arterial pressure variability as a marker of sympatho-vagal interaction in man and in conscious dog. Circulation Research 198659 178-193.

24 Pomeranz B, MacAulay RJB, Caudill MA, Kutz I, Adam D, Gordon D et al. Assesment of autonomic function in humans by heart rate spectral analysis. American Journal of Physiology. Heart and Circulatory Physiology 1985248 H151-H153.

25 Bernardi L, Leuzzi S, Radaelli A, Passino C, Johnston JA \& Sleight P. Low-frequency spontaneous fluctuations of $\mathrm{R}-\mathrm{R}$ interval and blood pressure in conscious humans: a baroreceptor or central phenomenon? Clinical Science 199487 649-654.

26 Matsukawa T, Mano T, Gotoh E, Minamisawa K \& Ishii M. Altered muscle sympathetic nerve activity in hyperthyroidism and hypothyroidism. Journal of the Autonomic Nervous System $199323207-211$.

27 Hammond HK, White FC, Buxton ILO, Saltzstein P, Brunton LL \& Longhurst JC. Increased myocardial $\beta$-receptors and adrenergic responses in hyperthyroid pigs. American Journal of Physiology. Heart and Circulatory Physiology 1987252 H283-H290.

28 Levey GS \& Klein I. Catecholamine-thyroid hormone interactions and the cardiovascular manifestation of hyperthyroidism. American Journal of Medicine $1990 \mathbf{8 8} 642-646$.

29 Sawin CT, Geller A, Wolf PA, Baker E, Bacharach P, Wilson PW et al. Low serum thyrotropin concentrations as a risk factor for atrial fibrillation in older persons. New England Journal of Medicine 1994331 1249-1252.

30 Biondi B, Palmieri EA, Fazio S, Cosco C, Nocera M, Sacca L et al. Endogenous subclinical hyperthyroidism affects quality of life and cardiac morphology and function in young and middle-aged patients. Journal of Clinical Endocrinology and Metabolism $2000 \mathbf{8 5}$ 4701-4705.

Received 26 April 2001

Accepted 26 July 2001 